\title{
Formation, Growth, and Destruction of Nuclear Star Clusters
}

\author{
Torsten Böker \\ European Space Agency, Keplerlaan 1, 2200AG Noordwijk, Netherlands \\ email: tboeker@rssd.esa.int
}

\begin{abstract}
This talk is an attempt to combine recent insights into the nature of the nuclear star clusters in galaxies of various morphologies into a coherent (albeit simplistic) picture for their formation, growth, and eventual destruction.
\end{abstract}

Keywords. galaxies: nuclei; galaxies: star clusters

\section{Formation and Growth of Nuclear Star Clusters}

The structural properties, masses, and stellar populations of nuclear star clusters (NSCs) have been extensively discussed elsewhere, both for late-type spirals (Böker et al. 2004, Walcher et al. 2005, 2006) as well as for spheroidal galaxies (Côté et al. 2006). A key finding from these studies is that, in general, the star formation histories of NSCs are long and complex, but only NSCs in late-type spirals also contain a young (few hundred Myrs) stellar population. The NSCs in spheroidal galaxies, on the other hand, generally have luminosity-weighted ages of many Gyrs, but are on average 3.5 Gyrs younger than the bodies of their host galaxies (Paudel 2010).

This implies that in the present-day universe, any growth mechanism that causes the "rejuvenation" of NSCs occurs only in (gas-rich) disk galaxies. The NSCs in (gas-poor) spheroidals, on the other hand, must have evolved passively for at least a few Gyrs. If they have acquired stellar mass over this timescale, it can only have occurred via the accretion of evolved stars, e.g. through the merging of globular clusters onto the NSC.

The merging of star clusters is indeed one of the proposed mechanisms for the build-up of NSCs (Capuzzo-Dolcetta \& Miocchi 2008). At least in disk galaxies, however, this is unlikely to be the whole story, because Hartmann et al. (2011) show that cluster infall alone cannot explain the dynamical properties of the NSC in the nearby edge-on disk NGC 4244 . They conclude that at least $50 \%$ of its mass must have been produced by in-situ star formation. The star formation is the result of gas infall into the central few pc, which can be caused by a number of mechanisms such as bar-induced torques (Schinnerer et al. 2006), compressive tidal forces (Emsellem \& van der Ven 2008), or the magneto-rotational instability in galaxy disks (Milosavljevic 2004).

In any case, it is important to keep in mind that the formation of NSCs and their subsequent growth do not necessarily have to be governed by the same mechanism, and that more than one mechanism can contribute to the evolution of an NSC after its formation.

\section{SMBHs and the Destruction of Nuclear Star Clusters}

The co-evolution of NSCs and central super-massive black holes (SMBHs) is currently a very active field of research, triggered by the realization that both types of a central massive object $(\mathrm{CMO})$ can co-exist at the low end of the SMBH mass range 


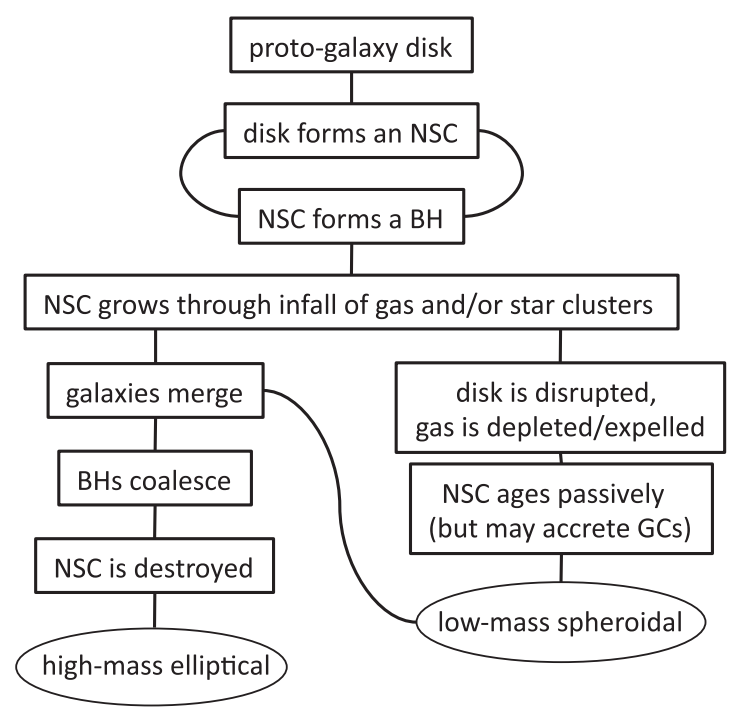

Figure 1. Possible evolutionary path of an NSC that forms early in the life of a gas-rich, disk-dominated "proto"-galaxy. As long as gas is available for infall into the center, the NSC (and any SMBH it may harbor) will grow. Once the gas is depleted or expelled (e.g. by the disruption of the galaxy disk through harassment or a close encounter), the CMO growth stops, and it will only evolve passively from then on. The host galaxy may progress along the merger tree, and its SMBH may well merge with the SMBHs of the merger partners, destroying their hosts' NSCs in the process.

$\left(M_{\mathrm{BH}}<10^{9} \mathrm{M}_{\odot}\right.$, Seth et al. 2008). Unfortunately, the exact mechanism for SMBH formation, and whether or not an NSC is necessary to form an SMBH remain unclear for now.

However, there appears to be a transition in what type of CMO dominates: at very low CMO masses, SMBHs are hard to identify (Satyapal et al. 2009), and their mass is usually less than that of the NSC. In galaxies hosting SMBHs with masses above $\approx 10^{10} \mathrm{M}_{\odot}$, on the other hand, NSCs are usually not observed (Graham \& Spitler 2009), suggesting that the most massive SMBHs have destroyed their host NSCs. A similar transition is also evident in the surface brightness profiles of spheroidal galaxies, which smoothly transition from a pronounced light excess in the central few pc of low-mass systems to a clear light deficit in high mass system (Côté et al. 2007). This gradual change may be identified with a decreasing prominence of the NSC as the mass of the host spheroid grows.

The mass ratio between SMBH and NSC may well be imprinted early-on in the galaxy's life by their mutual feedback during the "competitive accretion" phase, as proposed by Nayakshin, Wilkinson \& King (2009). However, it is also tempting to speculate that, as the evolution of the host galaxy progresses, the SMBH grows in mass at the expense of the NSC. For example, the merger of two BHs has been shown to efficiently destroy the surrounding NSC(s) via loss-cone depletion (e.g. Merritt 2006). While many details of NSC evolution remain to be addressed, the general picture outlined in Figure 1 appears to be consistent with what we currently know.

\section{References}

Böker, T., et al. 2004, AJ, 127, 105

Capuzzo-Dolcetta, R. \& Miocchi, P. 2008, MNRAS, 388, 69

Côté, P., et al. 2006, ApJS, 165, 57

Côté, P., et al. 2007, ApJ, 671, 1456 
Emsellem, E. \& van der Ven, G. 2008, ApJ, 674, 653

Graham, A. W. \& Spitler, L. R. 2009, MNRAS, 397, 2148

Hartmann, M., et al. 2011, MNRAS, 418, 2697

Merritt, D. 2006, Reports on Progress in Physics, 69, 2513

Milosavljevic, M. 2004, ApJL, 605, 13

Nayakshin S., Wilkinson M. I., King A. 2009, MNRAS, 398, 54

Paudel, S., Lisker, T., \& Kuntschner, H. 2010, MNRAS, 413, 1764

Satyapal, S., et al. 2009, ApJ, 704, 439

Seth, A., et al. 2008, 678, 116

Schinnerer, E., et al. 2006, ApJ, 649, 181

Walcher, C. J., et al. 2005, ApJ, 618, 237

Walcher C. J., et al. 2006, ApJ, 649, 692 